\title{
Les Élections à l'Assemblée nord-irlandaise de 2016 et 2017 : contextes, résultats, perspectives
}

The 2016 and 2017 Elections to the Northern Irish Assembly: Contexts, Results

and Prospects

\section{Karine Bigand}

\section{(2) OpenEdition}

1 Journals

\section{Édition électronique}

URL : http://journals.openedition.org/rfcb/1568

DOI : $10.4000 / \mathrm{rfcb} .1568$

ISSN : 2429-4373

Éditeur

CRECIB - Centre de recherche et d'études en civilisation britannique

\section{Référence électronique}

Karine Bigand, «Les Élections à l'Assemblée nord-irlandaise de 2016 et 2017 : contextes, résultats, perspectives », Revue Française de Civilisation Britannique [En ligne], XXII-4 | 2017, mis en ligne le 15 novembre 2017, consulté le 01 mai 2019. URL : http://journals.openedition.org/rfcb/1568 ; DOI : $10.4000 /$ rfcb. 1568

Ce document a été généré automatiquement le 1 mai 2019.

\section{$\oplus \Theta \Theta$}

Revue française de civilisation britannique est mis à disposition selon les termes de la licence Creative Commons Attribution - Pas d'Utilisation Commerciale - Pas de Modification 4.0 International. 


\section{Les Élections à l'Assemblée nord- irlandaise de 2016 et 2017 : contextes, résultats, perspectives}

The 2016 and 2017 Elections to the Northern Irish Assembly: Contexts, Results and Prospects

Karine Bigand

\section{Introduction}

1 L'Irlande du Nord a connu, entre mai 2016 et juin 2017, une séquence politique forte et inédite : deux élections locales se sont succédées à Stormont en moins d'un an (mai 2016 et mars 2017), la région est restée plusieurs mois sans gouvernement à partir de janvier 2017, et les élections parlementaires anticipées de juin 2017 ont mis dans la lumière sur le Democratic Unionist Party (DUP), majoritaire en Irlande du Nord, dont dépend la courte majorité parlementaire dont Theresa May dispose désormais. La perspective du Brexit, génératrice d'incertitudes, a imbriqué les situations politiques locales et nationales et menace des équilibres socio-économiques précaires dans de nombreuses régions britanniques. En Irlande du Nord, où $56 \%$ des électeurs ont voté pour le maintien dans l'Union Européenne, elle a été un facteur de déstabilisation politique non négligeable, tant les conséquences sur l'économie, les relations Nord/Sud et le processus de paix sont aussi inconnues que redoutées ${ }^{1}$.

Quoique principalement centré sur les élections à l'assemblée nord-irlandaise de mai 2016, cet article ne peut faire l'économie de couvrir également les élections locales de mars 2017, afin d'en comparer les contextes, les résultats et les perspectives.

Même si l'élection parlementaire de juin 2017 a donné un coup de projecteur inattendu sur les acteurs politiques nord-irlandais, quelques rappels s'imposent. Nous le savons, la scène politique est dominée par des clivages ethno-nationaux hérités de l'histoire du peuplement et des relations irlando-britanniques, la principale ligne de faille ayant trait 
au futur constitutionnel du territoire. Les unionistes/loyalistes sont partisans du maintien de l'Irlande du Nord au sein du Royaume-Uni, tandis que les nationalistes/ républicains sont en faveur d'une réunification de l'Irlande. Ce clivage fondamental se superpose à un clivage religieux, les unionistes/loyalistes étant majoritairement protestants, les nationalistes/républicains majoritairement catholiques : on parle, de fait, de communautés PUL ou CNR. Ces communautés sont chacune représentées par deux partis : le DUP, déjà cité, et le Ulster Unionist Party (UUP) pour les PUL, Sinn Féin et le Social Democratic Labour Party (SDLP) pour les CNR, le second parti de chaque duo étant plus modéré et accommodant envers l'autre communauté que le premier. Cette position de conciliation a permis à John Hume et David Trimble, respectivement leaders du SDLP et du UUP dans les années 1990, d'obtenir conjointement le Prix Nobel de la Paix en 1998 au sortir des négociations de paix qui aboutirent à la signature de l'Accord de Belfast en avril de la même année, mais elle n'a pas servi durablement leurs partis. Ainsi, depuis 2003, le DUP et Sinn Féin arrivent en tête des suffrages dans toutes les élections, qu'elles soient locales, nationales ou européennes. De ce point de vue, les élections de 2016 et 2017 pour l'Assemblée locale sont dans la continuité des trois précédentes.

4 Le seul prisme PUL/CNR n'est toutefois pas suffisant pour décrire la réalité du paysage ethno-national et politique nord-irlandais. D'une part, l'enquête Northern Ireland Life and Times, qui interroge régulièrement, depuis 1998, les habitants d'Irlande du Nord sur leurs attitudes et valeurs socio-politiques montre que plus de $40 \%$ de la population ne se définit ni comme unioniste, ni comme nationaliste : les résultats oscillent entre 40 et $47 \%$ sur la période 2007-2015 ${ }^{2}$. D'autre part, des partis non-communautaires existent et sont représentés à l'Assemblée locale, le plus ancien et le plus établi étant le parti de l'Alliance. Ces partis restent toutefois minoritaires: le parti écologiste avait un siège dans l'Assemblée sortante en mai 2016. Il y a aussi un certain nombre de membres sans étiquette. En outre, le paysage politique nord-irlandais est très largement distinct de son voisin britannique. Les partis d'envergure nationale y sont peu présents ou absents. Entre 2011 et 2016, seul le UKIP a pu se prévaloir d'un siège à l'Assemblée locale, après qu'un député UUP a changé de parti. Aux élections de 2016 et 2017, le parti conservateur, le UKIP et la branche nord-irlandaise du parti travailliste ont présenté des candidats, mais aucun n'a été élu.

Les élections à l'Assemblée locale du 5 mai 2016 étaient les cinquièmes depuis la création de l'assemblée nord-irlandaise en 1998, celles du 2 mars 2017 les sixièmes. L'assemblée et le gouvernement ont connu des débuts difficiles, ont été suspendues plusieurs fois entre 1998 et 2007, mais elles ont fonctionné sans discontinuer entre 2007 et janvier 2017. L'Accord de Belfast de 1998 a instauré un système dit " consociationnel », qui implique, d'une part, que les membres de l'Assemblée législative (MLA) se déclarent comme Nationalist/Unionist/Other au moment de leur investiture et, d'autre part, que l'exécutif repose sur le partage du pouvoir entre les partis ayant la plus grande assise électorale. Les partis qui disposent d'une représentation significative au sein de l'Assemblée peuvent prétendre à un portefeuille ministériel, la répartition entre les partis se faisant selon la méthode d'Hondt. Ce dispositif a pour but d'assurer la représentation du plus grand nombre de sensibilités possible au sein de l'exécutif et de forcer la coopération, et donc le compromis, entre partis.

6 Toutefois, au fil des ans, ce système de coalition obligatoire a fait l'objet de critiques: notamment parce qu'il est fondé sur et contribue à renforcer, par un phénomène de surenchère politique, les clivages ethno-nationaux dont la société nord-irlandaise veut 
sortir, mais aussi parce que l'implication de la majorité des partis dans l'exécutif laisse peu de place au nécessaire examen critique de son action ${ }^{3}$. Par ailleurs, la recherche constante d'un dénominateur commun ralentit la prise de décision et limite les ambitions politiques. Le risque d'impasse est constant, non seulement en raison de divergences idéologiques, mais également parce que certains dispositifs mis en place pour protéger les intérêts des différentes communautés sont parfois utilisés de manière abusive, ce qui entrave le processus politique. Chaque élection permet de constater le travail accompli et restant à accomplir pour atteindre un système normalisé. La tenue, à dix mois d'écart, de deux élections locales, suggère un système en stagnation. La comparaison des contextes et des résultats de ces deux moments politiques montre aussi, de fait, l'existence de certaines dynamiques.

\section{Contextes électoraux : I'enlisement du processus politique}

7 La législature 2011-2016 illustre les limites qui peuvent être reprochées au système consociationnel. Peu avant les élections de mai 2016, le gouvernement considérait avoir mis en place 64 des 82 mesures annoncées au début de la législature, mais l'agence de journalisme d'investigation The Detail montrait, dans une enquête indépendante, que seules 46 de ces mesures étaient effectives. 18 autres n'étaient que partiellement en place, les dernières n'ayant pas été abordées ${ }^{4}$.

De fait, deux raisons principales ont ralenti plusieurs fois le travail des institutions nordirlandaises au cours de la législature : la réforme du système de protection sociale liée aux restrictions budgétaires décidées par le gouvernement britannique depuis 2010, ainsi que les controverses concernant les défilés communautaires et l'utilisation des drapeaux dans l'espace public. A trois reprises, des négociations de plusieurs semaines sur ces points ont interrompu le travail de l'Assemblée sur les questions quotidiennes d'ordre socioéconomique («bread-and-butter issues »). En décembre 2013, des pourparlers menés par le diplomate américain Richard Haass furent un échec. En décembre 2014, de nouvelles négociations multipartites, dirigées par Teresa Villiers, alors Secrétaire d'Etat chargée de l'Irlande du Nord, avec le soutien du Premier Ministre britannique et son homologue irlandais, aboutirent à l'Accord de Stormont House (Stormont House Agreement) ${ }^{5}$. Cet accord a transféré aux institutions locales la responsabilité de la réforme du système de protection sociale, avec un coup de pouce financier de Londres si celle-ci était mise en place rapidement. Etait également actée la dévolution de pouvoir sur le taux d'imposition sur les sociétés (corporation tax) dans le but de rendre, à terme, l'Irlande du Nord plus attrayante pour les entreprises étrangères. Une partie de l'accord portait sur les éléments identitaires et le passé et prévoyait la création d'une série d'instances visant à faciliter le travail en commun sur ces questions sensibles. Enfin, la modernisation des institutions locales était envisagée, notamment la réduction du nombre de sièges par circonscription à l'horizon 2021 (de 6 à 5, soit 90 MLA au lieu de 108), ainsi que la possibilité pour les partis politiques de sortir du modèle de coalition obligatoire en rendant possible la constitution d'une opposition officielle. Ces modifications ont rapidement été effectives après l'élection de 2016, le UUP et le SDLP choisissant de constituer une opposition au gouvernement DUP/SF, tandis que les élections de 2017 ont effectivement désigné 90 membres à l'assemblée locale. Si bon nombre des mesures signalaient un processus de normalisation politique, la mise en application des décisions prises était souvent reportée 
à une échéance ultérieure, laissant le champ libre à d'autres développements susceptibles d'enrayer à nouveau la machine politique.

De fait, une nouvelle crise surgit à la fin de l'été 2015, après qu'une série de meurtres est attribuée à l'IRA et que le chef des services de police admet que l'organisation paramilitaire n'a pas cessé toutes ses activités. Après une crise gouvernementale et dix semaines de discussion, soit fin novembre 2015, le DUP et Sinn Féin s'accordent sur un «nouveau départ » («Fresh Start»), qui précise certains aspects de l'Accord de Stormont House $^{6}$. En particulier, la responsabilité de la réforme du système de protection sociale est renvoyée à Londres ${ }^{7}$, tandis que la réduction prévue du taux d'imposition sur les sociétés devra être compensée localement et non par une subvention de Londres. L'attachement à l'Etat de droit pour tous est réaffirmé et la promesse faite de réfléchir à une stratégie pour démanteler définitivement les groupes paramilitaires. Des échéances plus précises sont fixées, notamment en ce qui concerne les drapeaux et les défilés communautaires. Les réformes institutionnelles annoncées sont confirmées. La mise en place des initiatives liées à la difficile question de la gestion du passé (dealing with the past) reste, quant à elle, moins clairement définie ${ }^{8}$. Pour certains observateurs, notamment les groupes de victimes, ce manquement fait de ce nouveau départ un faux départ ${ }^{9}$.

Cette séquence, entre décembre 2013 et novembre 2015, ne représente pas l'intégralité du travail effectué par l'Assemblée de Stormont au cours de la dernière législature, mais reste symptomatique de la lenteur et de la difficulté du processus politique en Irlande du Nord. Elle est comparable à la séquence qui a mené à l'organisation d'élections anticipées en mars 2017. Au début du mois de janvier 2017, Martin McGuinness, Vice-Premier Ministre depuis 2007, démissionne de ses fonctions, sans que Sinn Féin ne lui désigne un remplaçant : ceci revient à mettre un terme au mandat de Première Ministre d'Arlene Foster, leader du DUP, les deux fonctions étant liées selon les termes de l'accord de 1998. La décision de McGuinness et de Sinn Féin fait suite au refus de Foster de se mettre temporairement en retrait du pouvoir, le temps qu'une enquête fasse la lumière sur des soupçons de malversations dans le cadre d'un programme de subventions aux énergies renouvelables (Renewable Heat Incentive) mis en place en 2012, alors qu'elle était ministre du commerce et de l'industrie. McGuinness dénonce aussi, entre autres, le manque de respect du DUP envers les communautés CNR, après que le ministre pour les communautés (DUP) a annulé la subvention annuelle de 50,000£ à des programmes favorisant l'apprentissage de la langue irlandaise ${ }^{10}$. Suite à sa démission, le processus est enclenché pour l'organisation de nouvelles élections locales, le 2 mars 2017.

\section{Les programmes électoraux ${ }^{11}$}

11 Le contexte nord-irlandais, contraint tant par les clivages identitaires que les rivalités inter- et intra-communautaires et le fonctionnement des institutions, laisse peu de place à l'audace dans les promesses électorales. Ainsi, l'analyse des programmes pour l'élection de 2016 révèle une grande convergence entre les cinq partis principaux (ceux ayant eu des responsabilités ministérielles), notamment en matière économique. Face au contexte socio-économique difficile et aux politiques d'austérité des deux gouvernements Cameron, tous les partis se déclarent pour la limitation des restrictions budgétaires, la modernisation des infrastructures et des transports, la construction de logements et la création d'emplois, en particulier dans l'industrie. Les cinq principaux partis déclarent aussi vouloir profiter de la responsabilité dévolue à Stormont quant à l'impôt sur les 
sociétés à compter de 2018. L'ambition affichée est de baisser le taux d'imposition, à l'instar de la République d'Irlande, afin de relancer les entreprises locales et d'attirer des entreprises étrangères en Irlande du Nord. Seuls le parti écologiste et le parti antiaustérité People Before Profit Association (PBPA) dénoncent les conséquences prévisibles d'un taux d'imposition bas sur les sociétés en termes de pression fiscale accrue sur les ménages.

Les lignes de fracture apparaissent sur les questions sociétales et sociales. Le modèle éducatif à adopter ne fait pas l'unanimité. Le DUP défend la sélection scolaire pour l'entrée dans le secondaire, tout comme le UUP, qui l'assortit toutefois d'investissements supplémentaires dans les écoles en difficulté. Le SDLP demande l'ouverture d'un débat sur la question, tandis que Sinn Féin et l'Alliance prônent la fin de la sélection. Le UUP, le SDLP et l'Alliance militent pour un système d'éducation intégré, où les enfants des communautés PUL et CNR seraient éduqués ensemble. Sinn Féin parle à la fois d'éducation intégrée et d'éducation partagée, modèle dans lequel des écoles confessionnelles partagent des équipements ou bâtiments, ce qui permet aux élèves de se rencontrer. Seule l'éducation partagée trouve sa place dans le programme du DUP. Sinn Féin met aussi l'accent sur l'éducation en langue irlandaise, qui réduit nécessairement les chances de mixité.

Les autres divergences principales qui se font jour, au-delà de la question constitutionnelle récurrente, portent sur les droits des minorités (ethniques et LGBT). Le SDLP, Sinn Féin et l'Alliance ont inscrit le mariage homosexuel dans leur programme, après qu'il a été adopté par référendum en République d'Irlande en mai 2015 et qu'une motion sur la question a reçu un vote favorable à Stormont en novembre 2015, après quatre refus depuis 2011. L'ensemble des partis, sauf le DUP qui ne mentionne pas ces questions, soutient la mise en place de stratégies sur l'égalité raciale et l'orientation sexuelle.

Le fonctionnement institutionnel n'est sans doute pas étranger à ces grands pans de consensus. L'accord de Stormont House de 2014 a affiné le fonctionnement de la coalition. Désormais, les partis ayant un nombre de sièges suffisant pour prétendre à un portefeuille ministériel doivent s'accorder sur l'établissement d'un Programme de Gouvernement. Si l'accord est général, une coalition est formée. Si certains partis sont en désaccord avec le contenu du programme, ils peuvent renoncer à prendre part à l'exécutif et créer une opposition officielle. Ce dispositif, mis en application par l'Assembly and Executive Reform (Assembly Opposition) Act (Northern Ireland) votée au début de l'année 2016, peut expliquer la convergence partielle des programmes du DUP et de Sinn Féin, quasi-certains de détenir la majorité des portefeuilles gouvernementaux ${ }^{12}$. Les autres partis, selon qu'ils envisagent de faire partie ou non de l'exécutif, doivent proposer des programmes complets et cohérents - on remarque ainsi que les programmes du UUP, du SDLP et de l'Alliance sont bien plus longs que ceux du DUP et de Sinn Féin, ce dernier présentant le plus court programme des cinq partis principaux (24 pages, dont la moitié en irlandais, contre 88 pages pour l'Alliance). Ce système de fonctionnement laisse aux petits partis ayant peu de chances de siéger au gouvernement le champ libre pour des propositions moins consensuelles. Ainsi, PBPA et le parti écologiste sont les seuls à demander l'extension à l'Irlande du Nord de la loi sur l'avortement de 1967, en vigueur en Grande-Bretagne.

15 L'essentiel des programmes est repris lors de la campagne pour les élections de 2017. Les lignes de faille entre les partis se creusent sur deux questions : le mariage homosexuel et 
les conséquences du Brexit. Hormis le DUP, tous les partis principaux se déclarent en faveur du mariage homosexuel, en phase avec la majorité de la population nordirlandaise. La politique d'obstruction du DUP, qui a fait échouer les motions à ce sujet à plusieurs reprises grâce au dispositif de la "petition of concern ", est clairement dénoncée ${ }^{13}$ . Certains partis, en particulier l'Alliance, inscrivent également dans leur programme la réforme de cette procédure, afin d'en éviter l'utilisation abusive couramment pratiquée dans la législature précédente ${ }^{14}$. Concernant la perspective du Brexit, les partis restent sur les positions qu'ils avaient défendues pendant la campagne du référendum. Le DUP, partisan du retrait, saisit l'opportunité de l'élection de 2017 pour se déclarer le meilleur garant de l'Union et de l'unionisme. Les partis qui avaient fait campagne en faveur du maintien dans l'UE (Sinn Féin, Alliance, SDLP, parti écologiste) demandent que soit négocié un statut spécial pour l'Irlande du Nord qui maintiendrait les quatre libertés de circulation fondamentales. PBPA, qui avait fait campagne pour la sortie de l'UE qu'il considère comme trop libérale, s'oppose au rétablissement d'une frontière "dure » entre l'Irlande du Nord et la République. Plus que les détails des programmes, c'est l'ambiance de la campagne qui est à souligner, notamment l'hostilité ouverte entre les deux principaux partis. Sinn Féin fait campagne sur deux maîtres mots - égalité et probité qui désignent, en creux, ce qu'il reproche au DUP. Inversement, le DUP tente de rassembler les communautés PUL au delà de ses bases habituelles (et donc au détriment du UUP), en arguant que seul un vote en sa faveur peut renforcer l'unionisme.

\section{Analyse des résultats}

Pour les élections locales, le territoire nord-irlandais est divisé en 18 circonscriptions, également utilisées pour les élections parlementaires (Carte 1). Le système électoral en place est celui du scrutin à vote unique transférable (STV, Single Transferable Vote), où les électeurs peuvent classer les candidats par ordre de préférence. Au moment du dépouillement des bulletins, le quota permettant l'obtention d'un siège est calculé. Si le nombre de premiers choix en faveur d'un candidat est supérieur au quota, le candidat est élu. Un transfert de vote est alors opéré, en distribuant aux autres candidats et en fonction des deuxièmes choix exprimés, les bulletins plaçant les candidats élus en tête de liste mais au-delà du quota nécessaire. Cette manœuvre est répétée jusqu'à ce que tous les sièges soient pourvus. Si aucun candidat n'atteint le quota lors du premier décompte, le candidat avec le moins de premiers choix est éliminé et les bulletins l'ayant placé en tête sont distribués aux autres candidats, en fonction des deuxièmes choix exprimés. L'avantage de ce système est d'offrir un choix plus grand aux électeurs, leur permettant de choisir non seulement entre les partis mais aussi entre les candidats. Il assure une plus forte représentativité de l'électorat, en prenant en compte plus de $80 \%$ des voix exprimées dans une circonscription à 6 sièges ${ }^{15}$. Ce système est également utilisé en Irlande du Nord pour les élections européennes et les élections locales.

Lors des élections du 5 mai 2016, 108 MLA ont été élus, soit 6 par circonscription. Le 2 mars 2017, suite à la mise en place de la réforme électorale, ce sont 90 MLA qui ont été désignés, soit 5 par circonscription. 


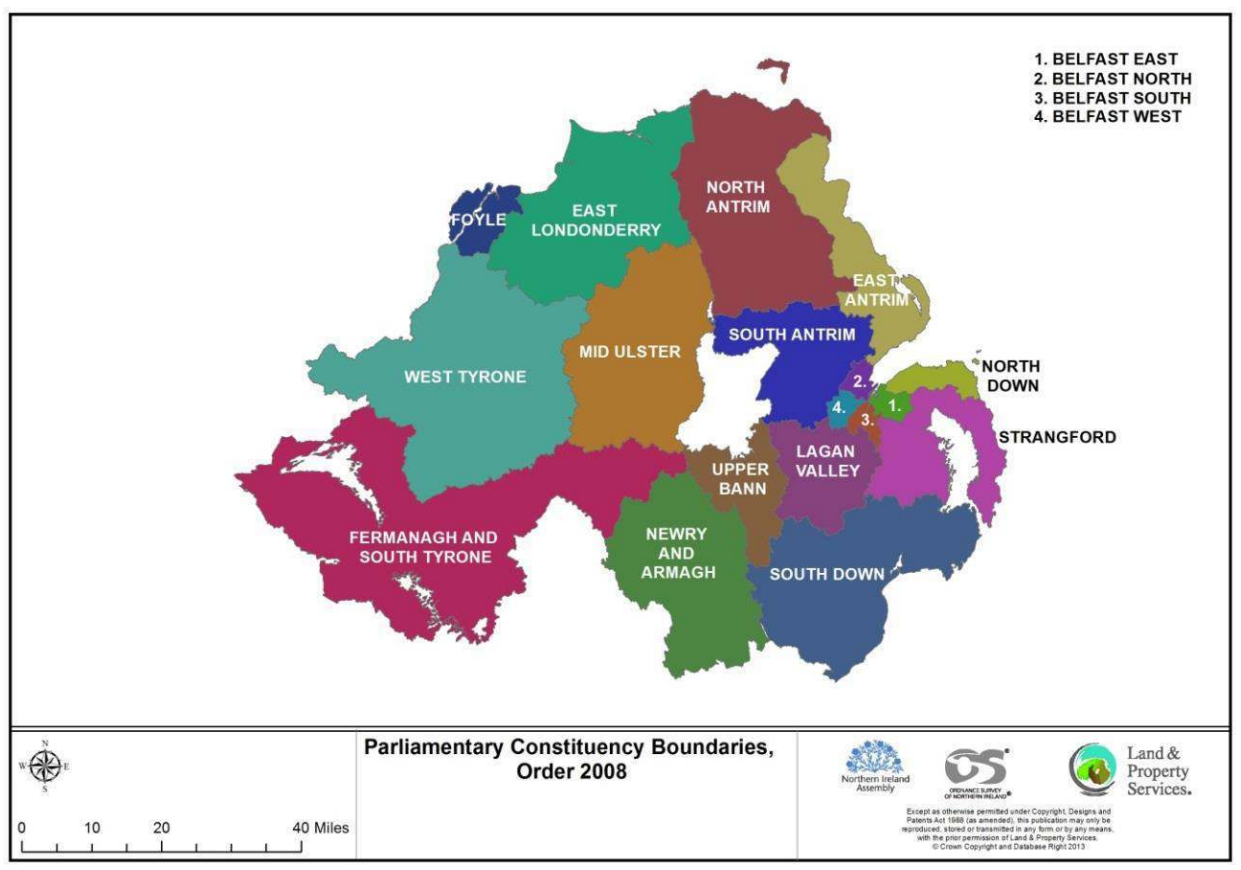

Carte 1. Circonscriptions électorales, Irlande du Nord'16

\section{Taux de participation}

Le Tableau 1 montre un regain du taux de participation aux élections de 2017 (64,8\%), après que les élections de 2016 ont atteint le niveau le plus bas depuis 1998 (54,9\%). La chute constante du taux de participation entre 1998 et 2016 est le signe d'un enthousiasme décroissant pour l'assemblée locale ${ }^{17}$. Dans ce contexte, la hausse de près de 10 points lors des élections de 2017 est tout à fait spectaculaire. Combinée aux résultats qui ont vu le DUP et Sinn Féin se renforcer, elle est le signe à la fois d'une mobilisation communautaire et d'un repli sur des positions ethno-nationales. Les 4 circonscriptions qui se sont le plus mobilisées sont les mêmes en 2016 et 2017, quoique dans des mesures différentes. Elles sont toutes situées à l'est et au sud de la province et sont à majorité CNR.

\begin{tabular}{|l|l|l|l|l|l|l|}
\hline \multirow{2}{*}{ Constituency } & 1998 & 2003 & 2007 & 2011 & 2016 & 2017 \\
\cline { 2 - 7 } & $\%$ & $\%$ & $\%$ & $\%$ & $\%$ & $\%$ \\
\hline Belfast East & 66.6 & 60.7 & 60.0 & 53.6 & 57.2 & 63.0 \\
\hline Belfast North & 67.3 & 62.3 & 60.9 & 50.3 & 52.5 & 61.8 \\
\hline Belfast South & 67.4 & 62.6 & 62.4 & 52.4 & 54.3 & 64.0 \\
\hline Belfast West & 70.5 & 65.9 & 67.4 & 57.9 & 57.8 & 66.8 \\
\hline East Antrim & 60.9 & 56.5 & 53.5 & 47.8 & 51.0 & 60.1 \\
\hline
\end{tabular}




\begin{tabular}{|l|c|c|c|c|c|c|}
\hline East Londonderry & 67.7 & 61.8 & 60.9 & 54.1 & 50.8 & 62.7 \\
\hline Fermanagh and South Tyrone & 79.4 & 72.9 & 71.2 & 69.0 & 64.6 & 72.6 \\
\hline Foyle & 72.0 & 63.5 & 63.9 & 57.8 & 56.0 & 65.0 \\
\hline Lagan Valley & 65.7 & 61.4 & 60.0 & 53.1 & 53.2 & 62.6 \\
\hline Mid Ulster & 84.4 & 74.9 & 73.1 & 65.4 & 58.8 & 72.4 \\
\hline Newry and Armagh & 77.3 & 70.2 & 70.8 & 61.3 & 59.3 & 69.4 \\
\hline North Antrim & 69.0 & 63.3 & 61.3 & 54.8 & 52.9 & 63.2 \\
\hline North Down & 60.2 & 54.5 & 53.8 & 45.9 & 49.6 & 59.2 \\
\hline South Antrim & 64.2 & 59.5 & 58.6 & 50.1 & 51.0 & 62.4 \\
\hline South Down & 73.7 & 65.6 & 65.0 & 58.1 & 53.8 & 66.2 \\
\hline Strangford & 61.6 & 57.1 & 54.5 & 48.6 & 50.3 & 60.9 \\
\hline Upper Bann & 72.3 & 64.2 & 61.1 & 55.3 & 54.4 & 62.5 \\
\hline West Tyrone & 79.4 & 73.2 & 71.7 & 64.0 & 59.9 & 69.9 \\
\hline Northern Ireland & 64.0 & 62.9 & 55.7 & 54.9 & 64.8 \\
\hline
\end{tabular}

Tableau 1 : Evolution du taux de participation aux élections pour l'Assemblée nord-irlandaise depuis $19988^{18}$

\section{Premiers choix des électeurs}

Le tableau 2 montre l'évolution de la répartition des premiers choix des électeurs depuis 1998. Si on compare les écarts entre le DUP et Sinn Féin depuis que ces deux partis sont en tête des suffrages, soit depuis 2003, on constate que l'élection de 2016 a produit l'écart le plus important entre les deux ( 5,2 points d'écart en faveur du DUP), tandis que celle de 2017 les place quasiment au coude à coude ( 0,2 point d'écart en faveur du DUP). De fait, l'élection de 2017, déclenchée par Sinn Féin pour dénoncer le comportement de son rival unioniste, lui a été favorable. Non seulement le parti a rattrapé le retard pris en 2016, mais il obtient son meilleur score depuis 1998. Le UUP et le SDLP se maintiennent dans des scores comparables, sans être en position de retrouver leur place prédominante de 1998. L'Alliance, premier parti non-communautaire, continue d'accroître sa popularité.

\begin{tabular}{|l|l|l|l|l|l|l|}
\hline Party & $\begin{array}{l}1998 \\
\%\end{array}$ & $\begin{array}{l}2003 \\
\%\end{array}$ & $\begin{array}{l}2007 \\
\%\end{array}$ & $\begin{array}{l}2011 \\
\%\end{array}$ & $\begin{array}{l}2016 \\
\%\end{array}$ & $\begin{array}{l}2017 \\
\%\end{array}$ \\
\hline DUP & 18.1 & 25.7 & 30.1 & 30.0 & 29.2 & 28.1 \\
\hline
\end{tabular}




\begin{tabular}{|l|l|l|l|l|l|l|}
\hline Sinn Féin & 17.6 & 23.5 & 26.2 & 26.9 & 24.0 & 27.9 \\
\hline UUP & 21.3 & 22.7 & 14.9 & 13.2 & 12.6 & 12.9 \\
\hline SDLP & 22.0 & 17.0 & 15.2 & 14.2 & 12.0 & 11.9 \\
\hline Alliance Party & 6.5 & 3.7 & 5.2 & 7.7 & 7.0 & 9.1 \\
\hline TUV & 2.5 & 3.4 & 2.6 & & & \\
\hline Progressive Unionist Party & 2.5 & 1.2 & 0.6 & 0.2 & 0.9 & 0.7 \\
\hline NI Workers' Council & 1.6 & 0.8 & & & & \\
\hline UK Unionist Party & 4.5 & 0.8 & 1.5 & & & \\
\hline Green & 0.1 & 0.4 & 1.7 & 0.9 & 2.7 & 2.3 \\
\hline PBPA & & & & 0.8 & 2.0 & 1.8 \\
\hline UKIP & 100.0 & 100.0 & 100.0 & 100.0 & 100.0 & 100.0 \\
\hline Independent/ Others & 5.8 & 4.2 & 4.5 & 3.0 & 4.8 & 2.5 \\
\hline Totals & & & & 0.6 & 1.5 & 0.2 \\
\hline
\end{tabular}

Tableau 2 : Répartition des premiers choix des électeurs, par parti (1998-2017) ${ }^{\mathbf{1 9}}$

\section{Répartition des sièges}

Il convient de comparer les élections de 2016 à celles de 2011, puis celles de 2017 à celles de 2016.

\begin{tabular}{|l|l|l|l|l|l|}
\hline \multirow{2}{*}{ Party } & \multirow{2}{*}{ Seats } & \multirow{2}{*}{$+/-$} & First Preference & Vote & \multirow{2}{*}{$+/-\%$} \\
\cline { 4 - 6 } & & & Votes & Share (\%) & \\
\hline DUP & 38 & & 202,567 & 29.2 & -0.8 \\
\hline Sinn Féin & 28 & -1 & 166,785 & 24.0 & -2.9 \\
\hline UUP & 16 & & 87,302 & 12.6 & -0.7 \\
\hline SDLP & 12 & -2 & 83,368 & 12.0 & -2.2 \\
\hline Alliance Party & 8 & & 48,447 & 7.0 & -0.7 \\
\hline TUV & 1 & & 23,776 & 3.4 & 0.9 \\
\hline Green & 2 & +1 & 18,718 & 2.7 & 1.8 \\
\hline
\end{tabular}




\begin{tabular}{|l|l|l|l|l|l|}
\hline PBPA & 2 & +2 & 13,761 & 2.0 & 1.2 \\
\hline UKIP & 0 & & 10,109 & 1.5 & 0.8 \\
\hline PUP & 0 & & 5,955 & 0.9 & 0.6 \\
\hline Conservative & 0 & & 2,554 & 0.4 & 0.4 \\
\hline Independent & 1 & & 22,65 & 3.3 & 0.9 \\
\hline Others & 0 & & 8,322 & 1.2 & \\
\hline Total & 108 & & 694,314 & 100.0 & 0.9 \\
\hline
\end{tabular}

Tableau 3 : Nombre de sièges et premiers choix exprimés, mai $2016^{20}$.

21 A l'issue du scrutin de mai 2016, les résultats en termes de nombre de sièges donnent l'image d'une grande stabilité (tableau 3). 276 candidats se sont présentés : 15 étaient sans étiquette, les autres représentaient 19 partis différents. Malgré cette diversité, le seul parti à faire son entrée sur la scène de Stormont est le parti anti-austérité PBPA. Les autres sièges sont allés aux sept partis ayant gagné des sièges en 2011. La hiérarchie des cinq partis ayant eu des responsabilités ministérielles entre 2011 et 2016 reste la même, trois d'entre eux (DUP, UUP, Alliance) gardant exactement le même nombre de sièges. Les deux partis perdants sont Sinn Féin et le SDLP, avec respectivement un et deux sièges perdus. En termes de proportion des premiers choix exprimés, on constate que les cinq partis ayant participé au gouvernement dans la dernière législature ont vu leur popularité baisser. L'érosion est plus nette (plus de 2 points, presque 3 pour Sinn Féin) pour les partis nationalistes, ce qui confirme la tendance baissière constatée lors des élections parlementaires de 2015 et des élections locales de 2014.

L'assemblée élue en 2016 est celle où la proportion des voix pour les partis non communautaires est la plus forte depuis la création des institutions en 1998. La proportion combinée des voix pour les deux plus grands partis (DUP et SF) est en recul par rapport aux deux élections précédentes (53,2\% en 2016 contre 56,9 en 2011 et 56,3\% en 2007), ce qui suggère, à tout le moins, un assouplissement dans les choix électoraux, voire une frustration grandissante à l'encontre des deux partis principaux.

\begin{tabular}{|c|c|c|c|c|c|c|c|c|c|}
\hline \multicolumn{10}{|c|}{ Seats by party and constituency (net change compared to $2011+/-$ ) } \\
\hline Parliamentary constituency & DUP & UUP & SF & SDLP & APNI & TUV & PBPA & Green & IND \\
\hline Belfast East & 3 & 1 & & & 2 & & & & \\
\hline Belfast North & 3 & & 2 & 1 & & & & & \\
\hline Belfast South & $2(+1)$ & $\begin{array}{l}0 \\
(-1)\end{array}$ & 1 & $\begin{array}{l}1 \\
(-1)\end{array}$ & 1 & & & $1(+1)$ & \\
\hline Belfast West & & & $\begin{array}{l}4 \\
(-1)\end{array}$ & 1 & & & $1(+1)$ & & \\
\hline
\end{tabular}




\begin{tabular}{|c|c|c|c|c|c|c|c|c|c|}
\hline East Antrim & 3 & 1 & 1 & & 1 & & & & \\
\hline East Londonderry & 3 & & 1 & 1 & & & & & 1 \\
\hline Fermanagh and South Tyrone & 2 & 1 & $\begin{array}{l}2 \\
(-1)\end{array}$ & $1(+1)$ & & & & & \\
\hline Foyle & 1 & & 2 & $2(-1)$ & & & $1(+1)$ & & \\
\hline Lagan Valley & $\begin{array}{l}3 \\
(-1)\end{array}$ & $2(+1)$ & & & 1 & & & & \\
\hline Mid-Ulster & 1 & 1 & 3 & 1 & & & & & \\
\hline Newry and Armagh & 1 & 1 & 3 & 1 & & & & & \\
\hline North Antrim & 3 & 1 & 1 & & & 1 & & & \\
\hline North Down & 3 & 1 & & & 1 & & & 1 & \\
\hline South Antrim & 3 & 1 & 1 & & 1 & & & & \\
\hline South Down & 1 & 1 & 2 & 2 & & & & & \\
\hline Strangford & 3 & 2 & & & 1 & & & & \\
\hline Upper Bann & 2 & 2 & $2(+1)$ & $\begin{array}{l}0 \\
(-1)\end{array}$ & & & & & \\
\hline West Tyrone & 1 & 1 & 3 & 1 & & & & & \\
\hline Northern Ireland & 38 & 16 & $28(-1)$ & $12(-2)$ & 8 & 1 & $2(+2)$ & $2(+1)$ & 1 \\
\hline
\end{tabular}

Tableau 4 : Répartition des sièges par circonscription, mai 2016 (comparaison avec 2011)21.

Le tableau de la répartition des sièges par circonscription (Tableau 4) montre que les changements ne se sont pas effectués uniquement entre les deux partis nationalistes, le parti écologiste et PBPA. On constate que sept sièges ont changé d'étiquette partisane, dont quatre au sein de la même communauté: le DUP a perdu un siège dans la circonscription de Lagan Valley au profit de l'UUP, qui lui en a concédé un à Belfast South; Sinn Féin a concédé un siège au SDLP dans la circonscription de Fermanagh et South Tyrone mais lui en a repris un dans la circonscription d'Upper Bann. Les partis non-communautaires (Alliance, écologiste, PBPA) ont gagné 12 sièges dans 9 circonscriptions. Les gains par rapport à l'assemblée sortante ont eu lieu dans la circonscription de Belfast South, plutôt aisée et mixte, où le parti écologiste a gagné un siège aux dépens du SDLP. Les deux autres sièges sont ceux de PBPA, gagnés au détriment de Sinn Féin et du SDLP, respectivement à Belfast West et Foyle, deux circonscriptions majoritairement nationalistes et plutôt défavorisées.

Les résultats de 2017 (tableau 5) ne permettent pas une comparaison directe avec l'élection précédente dans la mesure où le nombre de sièges n'est pas le même. 228 
candidats se sont affrontés pour 90 sièges. 22 candidats étaient sans étiquette, les autres représentaient 14 partis différents. Au vu du nombre de sièges perdus, l'érosion a été la plus grande pour les partis unionistes, en particulier le DUP. Le grand vainqueur est Sinn Féin, qui est passé d'à peine $26 \%$ à $30 \%$ des sièges. De fait, le scrutin de 2017 a vu opérer un repositionnement de l'électorat en termes ethno-nationaux, puisque la proportion combinée de premiers choix attribués au deux partis principaux passe d'environ 370,000 à 450,000 votants (soit 56\%). L'Alliance est le seul parti non-communautaire dont la proportion de premiers choix augmente.

\begin{tabular}{|l|l|l|l|l|l|}
\hline \multirow{2}{*}{ Party } & \multirow{2}{*}{ Seats } & + & First Preference & Vote & \multirow{2}{*}{ +/- \% } \\
\cline { 3 - 6 } & & & Votes & Share (\%) & \\
\hline DUP & 28 & -10 & 225,413 & 28.1 & -1.1 \\
\hline Sinn Féin & 27 & -1 & 224,245 & 27.9 & 3.9 \\
\hline UUP & 10 & -6 & 103,314 & 12.9 & 0.3 \\
\hline SDLP & 12 & -2 & 95,958 & 11.9 & -0.1 \\
\hline Alliance Party & 8 & & 72,717 & 9.1 & 2.1 \\
\hline TUV & 1 & & 20,523 & 2.6 & -0.9 \\
\hline Green & 2 & & 18,527 & 2.3 & -0.4 \\
\hline Independent & 1 & & 14,407 & 1.8 & -1.5 \\
\hline PBPA & 1 & -1 & 14,100 & 1.8 & -0.2 \\
\hline UKIP & 0 & & 10,109 & 1.5 & 0.8 \\
\hline PUP & 0 & & 5,590 & 0.7 & -0.2 \\
\hline Conservative & 0 & & 2,399 & 0.3 & -0.1 \\
\hline Others & 0 & & 6,122 & 0.8 & -1.6 \\
\hline Total & 90 & & 803,315 & 100.0 & \\
\hline
\end{tabular}

Tableau 5 : Nombre de sièges et premiers choix exprimés, mars $2017^{22}$.

Le changement majeur de l'élection de 2017 est que, pour la première fois depuis la création de l'Irlande du Nord, les partis unionistes n'ont pas la majorité absolue au parlement local. En 2016, le DUP, le UUP et le TUV cumulaient 55 sièges sur 108 (50,9\%) contre 39 sièges sur 90 en 2017 (43,3\%). Sinn Féin et le SDLP cumulaient 40 sièges sur 108 à l'issue du scrutin de 2016 (37\%) - ils en détiennent désormais 39 sur 90. Les deux traditions communautaires sont donc représentées à égalité, et les deux sont minoritaires. 
26 En raison de la réduction du nombre de sièges, l'analyse des résultats par circonscription est moins révélatrice que pour l'élection de 2016. C'est souvent le dernier candidat élu qui a perdu son siège. On note toutefois que l'Alliance et le parti écologiste maintiennent tous leurs sièges dans exactement les mêmes circonscriptions. En revanche, PBPA ne confirme pas sa percée. En 2016, le candidat PBPA Gerry Carroll était arrivé en tête des préférences des électeurs de la circonscription de Belfast West, fief traditionnel de Sinn Féin, avec 8299 voix (22,9\% des votes), soit plus de 3500 voix d'avance sur le premier candidat républicain. En 2017, Gerry Carroll est certes réélu, mais au troisième décompte des voix, et avec seulement 4,903 premiers choix en sa faveur. Un second siège PBPA était gagné en 2016 dans la circonscription de Foyle (Derry區Londonderry), par Eamonn McCann, figure locale majeure, militant historique pour les droits civiques, journaliste et syndicaliste. Ce siège est perdu en 2017.

\section{Un renouvellement politique?}

Les sièges remportés par le parti écologiste et le PBPA en 2016 pouvaient être considérés comme des frémissements dans un système politique apparemment stagnant. Créé en 2005, PBPA est un parti socialiste radical qui propose une alternative de gauche dans toute l'île d'Irlande, dans des systèmes politiques qui ne se fondent pas sur la distinction classique droite/gauche. Il monte en puissance depuis 2011 face aux programmes d'austérité mis en place en République d'Irlande et en Irlande du Nord. Ainsi, au sud de la frontière, PBPA compte 14 conseillers municipaux depuis 2014 et 3 membres du Dáil (parlement irlandais) depuis 2015. Le succès retentissant de Gerry Carroll en 2016, faisant suite à son élection au conseil municipal de Belfast en 2014, à l'âge de 27 ans, a souligné les limites des partis nationalistes traditionnels - notamment Sinn Féin, qui se définit comme socialiste - à continuer d'attirer les électeurs en situation de déclassement. L'élection de 2017 n'a pas confirmé ce frémissement, le contexte de clivage croissant avec le DUP et l'expérience de la machine électorale de Sinn Féin ayant permis de regagné le terrain perdu, mais une politique différente a pu être, même brièvement, entendue. Le parti a présenté 3 candidats en 2016, 7 en 2017.

D'autres évolutions sont à signaler, montrant l'émergence d'une nouvelle génération. Ainsi, le renouvellement des cadres politiques est encore plus flagrant dans l'assemblée de 2016 que dans la précédente, avec 31 membres nouvellement élus (28,7\%), contre 25 en $2011(23,1 \%)^{23}$. Alors que le député type était âgé de 52 ans en 2016 - donnée stable par rapport à l'assemblée sortante - 22 des nouveaux élus étaient en deçà de cette moyenne. Parmi les cinq principaux partis, c'est au sein du SDLP que le rajeunissement est le plus évident, puisque l'âge moyen des élus de ce parti, qui était de 50 en 2011, est de 44 ans en 2016. Le UUP, le SDLP et l'Alliance sont les partis dont les groupes se sont le plus renouvelés, avec respectivement 7,5 et 3 membres nouvellement élus sur un groupe parlementaire de 16, 12 et 8, signe de partis en transition. A l'inverse, le renouvellement est moindre pour DUP et Sinn Féin, avec chacun 6 membres nouvellement élus dans leurs contingents respectifs ${ }^{24}$. Le scrutin de 2017, intervenant à peine 10 mois plus tard, ne change pas ces tendances. 10 candidats y sont nouvellement élus.

La féminisation de l'Assemblée est également visible, tant en termes de candidates qu'en termes d'élues. Elle est le résultat d'une attitude proactive au sein de l'Assemblée quant à la sous-représentation des femmes en son sein, un phénomène flagrant par rapport au Parlement écossais et à l'Assemblée galloise ${ }^{25}$. Le taux de candidates est passé de 17,4\% en 
2011, à 27,5\% en 2016 et 30,7\% en 2017. Dans les deux élections, parmi les cinq principaux partis, le taux de candidates pour Sinn Féin était le plus largement au-dessus de la moyenne, tandis que celui du DUP en était le plus éloigné ${ }^{26}$. Les députées étaient au nombre de 30 en 2016 (27,8\%), contre 20 dans l'assemblée sortante (18,5\%). En 2017, elles sont 27 , soit $30 \%$ des MLA. Parmi les cinq principaux partis, c'est l'Alliance et Sinn Féin qui ont la plus importante proportion de MLA femmes (respectivement 40,7\% et 37,5\% en 2017), tandis que le DUP est à la traîne avec $21,4 \%$.

Enfin, le renouvellement est aussi celui des leaders politiques, puisque Arlene Foster (DUP) et Colum Eastwood (SDLP) ont pris la tête de leurs partis respectifs en 2015. Ce renouvellement s'est poursuivi en octobre 2016 par la nomination de Naomi Long, MLA pour Belfast East, à la tête de l'Alliance, à la suite de David Ford. L'année 2017 a prolongé ce renouvellement : Mike Nesbitt, né en 1957, MLA pour Strangford depuis 2011 et leader du UUP depuis 2012, a conservé son siège mais démissionné de la tête du parti suite au scrutin de mars. Il a été remplacé par Robin Swann, né en 1971 et MLA pour North Antrim. Gerry Adams reste le leader historique de Sinn Féin depuis 1983. Il a été MLA pour Belfast West de 1998 à 2010, avant d'être élu au Dáil en 2011. Son second, Martin McGuinness, a été MLA depuis 1998 (d'abord pour Mid-Ulster puis pour Foyle depuis 2016) et Vice-Premier Ministre de la province de 2007 à sa démission en janvier 2017. Il a été remplacé par Michelle O'Neill, née en 1977, et est décédé des suites d'une maladie le 21 mars 2017.

31 Ces données relatives aux profils des élus sont significatives. Données en contrepoint de l'impression d'enlisement du processus politique, elles signalent des partis politiques en mutation et une période de transition générationnelle dont les effets ne sont pas immédiatement visibles.

\section{Gouvernement et opposition, ou non}

Une fois les résultats proclamés, les partis élus sont tenus, selon les termes de l'Assembly and Executive Reform (Assembly Opposition) Act (Northern Ireland) de 2016, d'élaborer un Programme de Gouvernement et de constituer un gouvernement. Dans le système consociationnel en place, les deux partis unioniste et nationaliste majoritaires ont la prérogative de nommer respectivement le Premier Ministre et le Vice-Premier Ministre de la province. En mars 2016, Arlene Foster et Martin McGuinness ont été reconduits aux postes qu'ils occupaient avant l'élection. Hormis ces deux postes, le nombre de portefeuilles ministériels est désormais de 8 (contre 11 précédemment), ce qui veut dire que le nombre de sièges nécessaire pour prétendre à des responsabilités gouvernementales a augmenté, selon la méthode d'Hondt qui préside à leur attribution. Alors que 8 sièges suffisaient en 2011 pour pouvoir obtenir à un portefeuille, il en fallait 11 en 2016. Par conséquent, à l'issue de l'élection, seuls les partis communautaires étaient en mesure de siéger au gouvernement. L'Alliance a néanmoins participé à l'élaboration du Programme de Gouvernement avec les 4 autres partis, car il lui était possible de briguer le ministère de la Justice, dont l'attribution se fait non selon la méthode d'Hondt, mais après un vote des MLA, qui doit révéler un soutien des deux communautés. Selon les nouvelles dispositions, les partis qui le souhaitent peuvent, durant ou à la fin de l'élaboration du Programme de Gouvernement, renoncer à leurs prétentions ministérielles et former une opposition officielle. C'est ce qu'a choisi de faire le UUP, comme il l'avait annoncé à demimot dans son programme. Que le SDLP en fasse de même était moins prévisible. Dans 
leurs déclarations respectives, Mike Nesbitt et Colum Eastwood invoquent la même insatisfaction quant au Programme de Gouvernement, qu'ils estiment insuffisamment progressiste. Ils considèrent, en outre, qu'ils peuvent exercer les contrôles nécessaires sur l'action gouvernementale depuis les bancs de l'opposition ${ }^{27}$. La même logique a été adoptée par l'Alliance, qui a renoncé à présenter un candidat pour le Ministère de la Justice, trouvant le Programme de Gouvernement trop frileux. Le DUP et Sinn Féin se sont donc partagé les 7 ministères alloués par la méthode d'Hondt (4 pour le DUP, 3 pour Sinn Féin), tandis que Claire Sudgen, MLA sans étiquette, mais enregistrée en tant qu'unioniste, a bénéficié d'un soutien intercommunautaire suffisant pour être nommée Ministre de la Justice.

Cette situation post-électorale, qui voyait le UUP et le SDLP sur les bancs de l'opposition, était inédite. Au fil des mois, des rapprochements ont eu lieu entre les deux partis. En octobre 2016, Colum Eastwood a fait un discours au congrès annuel du UUP, reconnaissant des points de convergence et appelant de ses vœux une collaboration étroite entre les deux partis ${ }^{28}$. Durant la campagne des élections de mars 2017, les deux partis ont conseillé à leurs électeurs de faciliter le report de voix sur les candidats de l'autre parti, plutôt que vers Sinn Féin ou le DUP. Si cette stratégie d'élaboration de visions modérées et compatibles du nationalisme et de l'unionisme n'a pas payé, la nouvelle configuration politique constitue une forme de normalisation, en faisant de la place à une opposition. Dans ce contexte, le DUP et Sinn Féin font figure d'une hydre à deux têtes et sont critiqués, non plus seulement depuis l'extérieur de Stormont, mais aussi depuis les bancs de l'Assemblée. Toutefois, le rejet de ces deux partis n'est pas assez fort, en mars 2017, pour que les électeurs osent une alternative plus modérée, préférant se retrancher sur des partis dont ils pensent qu'ils ne transigeront pas avec leurs intérêts communautaires.

Depuis mars 2017, l'avenir de l'Irlande du Nord est incertain. La période de négociation normalement autorisée pour la formation d'un gouvernement a été étendue à de maintes reprises. Au début du mois de juillet 2017, alors que la saison des défilés communautaires approche, l'Irlande du Nord n'a toujours pas de gouvernement local. La tenue d'élections parlementaires nationales anticipées, décidée par Theresa May dans le but de consolider sa majorité à la Chambre des Communes, n'a fait que compliquer la situation davantage. Loin de gagner son pari, la Première Ministre se retrouve, à l'issue du scrutin du 8 juin 2017, à la tête d'un gouvernement conservateur minoritaire. Sa majorité dépend du soutien durable des dix députés DUP, négocié sous forme d'un milliard de livres de financement pour les services publics et l'infrastructure de la province. Cet accord est critiqué en Angleterre et dans les autres régions autonomes, car il va à l'encontre des principes fixant les modalités des dépenses publiques dans les différentes régions britanniques, le tout dans un contexte de restrictions budgétaires durables. Il est aussi perçu par divers commentateurs de tous bords politiques - John Major et Tony Blair notamment - comme un danger pour le processus de paix, qui exige que le gouvernement britannique soit un acteur neutre et ne favorise aucun parti local ${ }^{29}$. De nombreux points de blocage persistent entre le DUP et Sinn Féin, notamment sur la participation d'Arlene Foster au gouvernement, le mariage homosexuel, la promotion de la langue irlandaise, la défense des intérêts de la province dans la perspective du Brexit et les initiatives à mettre en place pour gérer l'héritage du conflit. à l'Irlande du Nord, James Brokenshire. L'option privilégiée jusqu'à présent a été de 
maintenir le dialogue entre les partis en prolongeant la période de négociations. Dans cette configuration, il revient au Secrétaire d'Etat de prendre les dispositions nécessaires pour que les services publics fonctionnent sans qu'un budget n'ait été voté. Une autre possibilité est d'organiser de nouvelles élections, afin de modifier les équilibres. Enfin, les institutions peuvent être officiellement suspendues et l'administration directe rétablie. Ces deux derniers scénarios ont un fort potentiel de déstabilisation, dans un contexte où l'Irlande du Nord et le gouvernement britannique sont particulièrement vulnérables. Pour certains commentateurs, la séquence récente illustre les limites du système consociationnel.

\section{Conclusion}

Nous avons évoqué en introduction le décalage entre, d'une part, le fonctionnement des institutions et le système des partis et, d'autre part, les attentes de l'électorat. Dans l'attente de la publication des études en cours sur les comportements électoraux lors de ces élections ${ }^{30}$, l'enquête Northern Ireland Life and Times signale des attitudes différentes selon les générations. En 2015, 40\% des personnes interrogées déclaraient ne se sentir ni unionistes ni nationalistes, dont $52 \%$ des $18-34$ ans et $49 \%$ des les $35-44$ ans $^{31}$. Pour ce qui est du soutien à un parti politique, $50 \%$ déclaraient se sentir le plus proche d'un des quatre partis communautaires, mais $24 \%$ ne se reconnaissaient dans aucun parti cité (y compris l'Alliance) ${ }^{32}$. Le soutien aux partis « verts et oranges » était plus faible parmi les plus jeunes, où c'était plutôt le rejet de tous les partis qui primait. L'avenir dira si ces générations parviendront à transformer le paysage politique nord-irlandais.

De fait, la marge de manœuvre vers une normalisation politique est conséquente. Des propositions de réformes institutionnelles, comme la refonte du dispositif de la «petition of concern ", la suppression des étiquettes communautaires et le passage à une majorité qualifiée de l'ordre des deux tiers pour l'adoption des lois, étaient présentes dans les programmes électoraux de ces deux élections, à défaut d'être effectivement débattues.

Ainsi, si les développements récents suggèrent l'enlisement, les frémissements démographiques et politiques sous-jacents laissent ouvertes les possibilités de reconfigurations, tant la situation de blocage actuel est intenable sur la durée.

Karine Bigand est Maître de Conférences en Etudes irlandaises à Aix-Marseille Université. Elle est membre du LERMA (Laboratoire d'Études et de Recherche sur le Monde Anglophone, EA853). Ses recherches portent sur les représentations et les usages publics de l'histoire sur l'île d'Irlande. Ses travaux récents s'intéressent au rôle des musées, en particulier dans l'Irlande du Nord post-conflit.

\section{BIBLIOGRAPHIE}

«A Fresh Start : The Stormont Agreement and Implementation Plan » https://www.gov.uk/ government/uploads/system/uploads/attachment_data/file/479116/A_Fresh_Start_- 
_The_Stormont_Agreement_and_Implementation_Plan_-_Final_VersionNov_2015_for_PDF.pdf Consulté le 10 novembre 2016.

«Stormont House Agreement », https://www.gov.uk/government/uploads/system/uploads/ attachment_data/file/390672/Stormont_House_Agreement.pdf Consulté le 10 novembre 2016 ASSEMbly AND EXECUTIVE REVIEW COMmitTEe, « Report on Women in Politics and the Northern Ireland Assembly », Belfast : Northern Irish Assembly, février 2015. URL : http:// www.niassembly.gov.uk/globalassets/documents/reports/assem_exec_review/women-inpolitics.pdf. Consulté le 10 novembre 2016.

BBC WEBSITE, «Victims Forum demands apology over Stormont deal omission », 19 novembre 2015. http://www.bbc.com/news/uk-northern-ireland-34875061. Consulté le 10 novembre 2016 BIGAND, Karine, «L'Irlande du Nord : un territoire vulnérable face au Brexit », Recherches internationales, vol. 109 (avril-juin 2017), p. 65-82.

BIRRELL, Derek, « Northern Ireland's Fresh Start Agreement Leaves Major Issues Unresolved », 18/01/16, Political Studies Association website. https://www.psa.ac.uk/insight-plus/blog/ northern-irelands-fresh-start-agreement-leaves-major-issues-unresolved Consulté le 10 novembre 2016.

DEVENPORT, Mark, « 2015 in review: Politics in Northern Ireland », BBC News, 29 décembre 2016. URL : http://www.bbc.com/news/uk-northern-ireland-35126563. Consulté le 10 novembre 2016. MCGARRY, John et O'LEARY, Brendan, « Power shared after the deaths of thousands ", in Rupert TAYLOR (ed.) Consociational Theory: McGarry and O'Leary and the Northern Ireland conflict, Routledge, 2009, p. 15-84

MCGLYNN Catherine, TONGE Jonathan et MCAUley Jim, « The Party Politics of Post-devolution identity in Northern Ireland », British Journal of Politics and International Relations, vol. 16, 2014, p. 273-290. DOI : 10.1111/j.1467-856X.2012.00528.x Consulté le 19 novembre 2016.

MCGUINNESS, Martin, lettre de démission, 9 janvier 2017. http://www.sinnfein.ie/files/2017/ Martin_McGuinnessResignationLetter.pdf MCKERRELL, Nick, « How international law could scupper a Tory deal with the DUP », The Conversation, 16 juin 2017. https://theconversation.com/how-international-law-could-scupper-atory-deal-with-the-dup-79583. Consulté le 3 juillet 2017.

NORTHERN IRELAND LIFE AND TIMES SURVEY, Political Attitudes Module, Question UNINATID. URL : http://www.ark.ac.uk/nilt/results/polatt.html\#identity. Consulté le 15 novembre 2016. NORTHERN IRELAND LIFE AND TIMES SURVEY, Political Attitudes Module, Question UNINATID 2015. URL : http://www.ark.ac.uk/nilt/2015/Political_Attitudes/UNINATID.html. Consulté le 15 novembre 2016.

NORTHERN IRELAND LIFE AND TIMES SURVEY, Political Attitudes Module, Question POLPART2. URL : http://www.ark.ac.uk/nilt/2015/Political_Attitudes/POLPART2.html. Consulté le 15 novembre 2016.

PEYRONEL, Valérie, « Northern Ireland: Devolution as an Electoral Issue in the 2015 UK General Election », Revue Française de Civilisation Britannique [Online], XX-3 | 2015, Online since 01 December 2015. URL : http://rfcb.revues.org/647 ; DOI : 10.4000/rfcb.647. Consulté le 20 octobre 2016.

RUSSELL, Raymond, « Research and Information Service Research Paper. Election Report: Northern Ireland Assembly Election, 2 March 2017 », Belfast, Northern Ireland Assembly, 8 mars 
2017. http://www.niassembly.gov.uk/globalassets/documents/raise/

publications/2017-2022/2017/general/2217.pdf Consulté le 3 juillet 2017.

RUSSELL, Raymond, « Research and Information Service Research Paper. Election Report:

Northern Ireland Assembly Election, 5 May 2016 », Belfast, Northern Ireland Assembly, 12 mai

2016. URL : http://www.niassembly.gov.uk/globalassets/documents/raise/

publications/2016-2021/2016/general/3616.pdf Consulté le 19 novembre 2016.

RUSSELL, Raymond, « Research and Information Service Research Paper. Northern Ireland Assembly Election 2011 », Belfast, Northern Ireland Assembly, 17 mai 2011. URL : http:// www.niassembly.gov.uk/globalassets/documents/raise/publications/2011/general/5511.pdf consulté le 19 novembre 2016.

SMYTH, Claire, «Stormont fails to deliver nearly half of its government commitments », The Detail, 27 avril 2016. URL : http://www.thedetail.tv/articles/stormont-fails-to-deliver-nearly-half-of-itsgovernment-commitments. Consulté le 15 novembre 2016.

SMYTH, Claire, « Stormont's petition of concern used 115 times in five years, The Detail, 29 septembre 2016. URL : http://www.thedetail.tv/articles/stormont-s-petition-of-concernused-115-times-in-five-years Consulté le 19 novembre 2016.

WILSON, Robin, Northern Ireland Peace Monitoring Report 4, Belfast, Community Relations Council, 2016, p. 167-168. URL : http://www.community-relations.org.uk/wp-content/uploads/2016/11/ Northern-Ireland-Peace-Monitoring-Report-2016.pdf Consulté le 10 novembre 2016.

\section{NOTES}

1. BIGAND, Karine, "L'Irlande du Nord: un territoire vulnérable face au Brexit ", Recherches internationales, vol. 109 (avril-juin 2017), p. 65-82.

2. NORTHERN IRELAND LIFE AND TIMES SURVEY, Political Attitudes Module, Question UNINATID. http://www.ark.ac.uk/nilt/results/polatt.html\#identity. Consulté le 15 novembre 2016.

3. MCGARRY, John et O'LEARY, Brendan, "Power shared after the deaths of thousands ", in Rupert TAYLOR (ed.) Consociational Theory: McGarry and O'Leary and the Northern Ireland conflict, Routledge, 2009, p. 15-84 ; MCGLYNN Catherine, TONGE Jonathan et MCAULEY Jim, « The Party Politics of Postdevolution identity in Northern Ireland», British Journal of Politics and International Relations, vol. 16, 2014, p. 274-276.

4. SMYTH, Claire, "Stormont fails to deliver nearly half of its government commitments », The Detail, 27 avril 2016. http://www.thedetail.tv/articles/stormont-fails-to-deliver-nearly-half-ofits-government-commitments. Consulté le 15 novembre 2016.

5. «Stormont House Agreement", https://www.gov.uk/government/uploads/system/uploads/ attachment_data/file/390672/Stormont_House_Agreement.pdf, consulté le 10 novembre 2016; PEYRONEL, Valérie, « Northern Ireland: Devolution as an Electoral Issue in the 2015 UK General Election», Revue Française de Civilisation Britannique, vol. xx-3, 2015, DOI : 10.4000/rfcb.647, c onsulté le 20 octobre 2016; wiLson, Robin, Northern Ireland Peace Monitoring Report 4, Belfast, Community Relations Council, 2016, p.167-8. http://www.community-relations.org.uk/wpcontent/uploads/2016/11/Northern-Ireland-Peace-Monitoring-Report-2016.pdf, consulté le 10 novembre 2016.

6. «A Fresh Start: The Stormont Agreement and Implementation Plan» https://www.gov.uk/ government/uploads/system/uploads/attachment_data/file/479116/A_Fresh_Start_-

_The_Stormont_Agreement_and_Implementation_Plan_-_Final_VersionNov_2015_for_PDF.pdf. Consulté le 10 novembre 2016. 
7. En mars 2015, Sinn Féin, en désaccord avec la réforme du système de protection sociale et trouvant que les garanties avancées par Londres pour compenser les réductions budgétaires étaient insuffisantes, avait mis son véto à la réforme, la renvoyant à nouveau dans une impasse. Voir la synthèse de l'année 2015 par Mark DEVENPORT : http://www.bbc.com/news/uk-northernireland-35126563. Consulté le 10 novembre 2016.

8. WILSON, Robin, op. cit., p. 168-169.

9. BBC WEBSITE, « Victims Forum demands apology over Stormont deal omission », 19 novembre 2015. http://www.bbc.com/news/uk-northern-ireland-34875061, consulté le 10 novembre 2016 ; BIRRELL, Derek, « Northern Ireland's Fresh Start Agreement Leaves Major Issues Unresolved ", 18/01/16, Political Studies Association website. https://www.psa.ac.uk/insight-plus/blog/ northern-irelands-fresh-start-agreement-leaves-major-issues-unresolved, consulté le 10 novembre 2016.

10. MCGUINNESS, Martin, lettre de démission, 9 janvier 2017. http://www.sinnfein.ie/files/2017/ Martin_McGuinnessResignationLetter.pdf. Consulté le 30 juin 2017.

11. Les programmes électoraux des principaux partis pour les deux élections sont téléchargeables à partir de cet URL: http://cain.ulst.ac.uk/issues/politics/election/ manifestos.htm. Consulté le 30 juin 2017.

12. Les deux partis s'accordent sur la création de 50000 emplois, l'investissement d'un milliard de livres dans le système de santé et un nombre de logements sociaux compris entre 8000 et 10 000 sur 5 ans.

13. Ce dispositif permet à un groupe de 30 députés d'un même bord politique (nationaliste ou unioniste) d'exiger qu'un soutien inter-communautaire (de $40 \%$ dans les deux communautés) soit apporté à une question, en plus d'une majorité qualifiée de $60 \%$ des membres présents et votants. Inclus dans l'Accord de 1998 afin de protéger les intérêts des communautés ethno-nationales, ce système est controversé car le vote des députés déclarés nationalistes ou unionistes compte davantage que celui des députés étiquetés Other. Il est aussi fréquemment utilisé comme véto sur des questions qui n'ont rien de communautaire. Ainsi, Sinn Féin l'a utilisé pour bloquer la réforme du système de protection sociale au printemps 2015 et le DUP pour bloquer la majorité (nationaliste et Other) favorable au mariage pour tous en novembre 2015.

14. SмYTH, Claire, "Stormont's petition of concern used 115 times in five years, The Detail, 29 septembre 2016. http://www.thedetail.tv/articles/stormont-s-petition-of-concern-used-115times-in-five-years. Consulté le 19 novembre 2016.

15. RUSSELL, Raymond, «Research and Information Service Research Paper. Election Report: Northern Ireland Assembly Election, 5 May 2016 », Belfast, Northern Ireland Assembly, 12 mai 2016, p. 6 . http://www.niassembly.gov.uk/globalassets/documents/raise/ publications/2016-2021/2016/general/3616.pdf, consulté le 19 novembre 2016.

16. RUSSELL, Raymond, «Research and Information Service Research Paper. Election Report: Northern Ireland Assembly Election, 5 May $2016 »$, op. cit., p. 7.

17. Voir à ce sujet les enquêtes et l'analyse proposées dans WILson, Robin, op. cit., p. 173-174.

18. RUSSELL, Raymond, «Research and Information Service Research Paper. Election Report: Northern Ireland Assembly Election, 2 March 2017 », Belfast, Northern Ireland Assembly, 8 mars 2017, p. $9 . \quad$ http://www.niassembly.gov.uk/globalassets/documents/raise/ publications/2017-2022/2017/general/2217.pdf. Consulté le 3 juillet 2017.

19. RUSSELL, Raymond, «Research and Information Service Research Paper. Election Report: Northern Ireland Assembly Election, 2 March $2017 »$, op. cit., p. 13.

20. RUSSELL, Raymond, «Research and Information Service Research Paper. Election Report: Northern Ireland Assembly Election, 5 May $2016 »$, op.cit., p. 11-12.

21. Adapté de RUSSELL, Raymond, «Research and Information Service Research Paper. Election Report: Northern Ireland Assembly Election, 5 May 2016 », op. cit., p. 14. 
22. RUSSELL, Raymond, "Research and Information Service Research Paper. Election Report: Northern Ireland Assembly Election, 2 March 2017 », op. cit., p. 11-12.

23. RUSSELL, Raymond, "Research and Information Service Research Paper. Election Report: Northern Ireland Assembly Election, 5 May 2016 », op. cit., p. 3 ; RUSSELL, Raymond, « Research and Information Service Research Paper. Northern Ireland Assembly Election 2011 », Belfast, Northern Ireland Assembly, p. 3. http://www.niassembly.gov.uk/globalassets/documents/raise/ publications/2011/general/5511.pdf. Consulté le 19 novembre 2016.

24. Statistiques générées à partir de http://aims.niassembly.gov.uk/mlas/membersmontage.aspx . Consulté le 19 novembre 2016.

25. ASSEMbly AND EXeCUtive ReVieW committee, « Report on Women in Politics and the Northern Ireland Assembly », Belfast: Northern Irish Assembly, février 2015. http:// www.niassembly.gov.uk/globalassets/documents/reports/assem_exec_review/women-inpolitics.pdf. Consulté le 10 novembre 2016.

26. RUSSELL, Raymond, «Research and Information Service Research Paper. Election Report: Northern Ireland Assembly Election, 5 May 2016 », op. cit., p. 8 ; RUSSELL, Raymond, « Research and Information Service Research Paper. Northern Ireland Assembly Election 2011 », op. cit., p. 9 ; RUSSELL, Raymond, «Research and Information Service Research Paper. Election Report: Northern Ireland Assembly Election, 2 March 2017 », op. cit., p. 7

27. Déclaration de Mike Nesbitt, 12 mai 2016, UUP website, http://uup.org/news/4255/NEWERA-FOR-DEVOLVED-POLITICS-AS-ULSTER-UNIONISTS-FORM-OFFICIAL-OPPOSITION-AT-

NORTHERN-IRELAND-ASSEMBLY\#.WDDaO1tri2w Consulté le 19 novembre 2016.

Déclaration de Colum Eastwood, «SDLP to form constructive opposition », 19 mai 2016, SDLP website, http://www.sdlp.ie/news/2016/sdlp-to-form-constructive-opposition/ Consulté le 19 novembre 2016.

28. SDLP website, "SDLP Leader addresses UUP Conference 2016 », 22 octobre 2016. http:// www.sdlp.ie/news/2016/sdlp-leader-addresses-uup-conference-2016/ Consulté le 19 novembre 2016.

29. MCKERRELL, Nick, "How international law could scupper a Tory deal with the DUP », The Conversation, 16 juin 2017. https://theconversation.com/how-international-law-could-scupper-atory-deal-with-the-dup-79583. Consulté le 3 juillet 2017.

30. Voir le projet « The Northern Ireland Assembly Election Study 2016 » à Queen's University, Belfast. http://www.qub.ac.uk/sites/NIAES2016/ Consulté le 15 novembre 2016.

31. NORTHERN IRELAND LIFE AND TIMES SURVEY, Political Attitudes Module, Question UNINATID 2015. http://www.ark.ac.uk/nilt/2015/Political_Attitudes/UNINATID.html. Consulté le 15 novembre 2016.

32. NORThern IRELAND Life AND times SURvey, Political Attitudes Module, Question POLPART2. http://www.ark.ac.uk/nilt/2015/Political_Attitudes/POLPART2.html. Consulté le 15 novembre 2016.

\section{RÉSUMÉS}

Les élections du 5 mai 2016 étaient les cinquièmes depuis la création de l'assemblée nordirlandaise en 1998. Si la mise en place des institutions décentralisées s'est faite dans la douleur, avec plusieurs interruptions dans les premières années de leur existence, elles n'ont pas connu 
de suspension officielle depuis 2007. Toutefois, près de 20 ans après l'Accord du Vendredi Saint, les structures internes des institutions et le paysage politique clivé propre à la province nuisent à la fluidité et à l'efficacité des débats et des prises de décision. Pour preuve, les élections anticipées organisées en mars 2017, dans un contexte de crise et d'hostilité entre les principaux partis. Ces deux scrutins sont l'occasion d'évaluer le chemin parcouru et restant à parcourir sur la voie de la normalisation. Là où les termes d'impasse ou d'enlisement sont fréquemment utilisés, à raison, pour décrire ce qui se passe sur la colline de Stormont, nous verrons que ces élections ont aussi vu émerger des tendances susceptibles de dynamiser, peut-être à moyen terme, un système en apparence stagnant.

The 2016 elections to the Northern Irish Assembly were the fifth since its creation in 1998 as part of the Belfast Agreement. As we know, the implementation of devolved institutions in Northern Ireland has not been plain sailing, and both the Assembly and the Executive were suspended several times. Although they have worked without official interruption since 2007, one cannot but observe that nearly 20 years into the post-conflict era, the internal structures of the institutions, as well as the quintessentially divided Northern Irish political landscape are detrimental to fluid and efficient debates and to the decision-making process - as is illustrated by the snap election called in March 2017, in a context of crisis and hostility between the two main parties. Both elections offer an opportunity to assess how far the road to normalisation has been travelled and remains to be travelled. Whereas words like stalemate and logjam are commonly and pointedly used to describe the work done on Stormont Hill, this article will show how the 2016 and 2017 elections have witnessed emerging new trends which might, possibly in the medium-term, kickstart a seemingly static system.

\section{INDEX}

Mots-clés : Stormont, Irlande du Nord, élections, dévolution

Keywords : Stormont, Northern Ireland, elections, devolution

\section{AUTEUR}

\section{KARINE BIGAND}

Aix Marseille Univ, LERMA, Aix-en-Provence, France. 